\title{
Expression of Vascular Endothelial Growth Factor (VEGF) Gene in Oral Squamous Cell Carcinoma
}

Edirisinghe $\mathrm{EAST}^{1}$, Weerasekera $\mathrm{MM}^{2}$, Yasawardene $\mathrm{SG}^{1}$

${ }^{\prime}$ Department of Anatomy, Faculty of Medical Sciences, University of Sri Jayewardenepura, SriLanka

${ }^{2}$ Department of Microbiology, Faculty of Medical Sciences, University of Sri Jayewardenepura, Sri Lanka

\section{Introduction}

Oral squamous cell carcinoma (OSCC) is the second most common cancer in Sri Lanka. Tumor growth and metastasis depend on angiogenesis and lymph angiogenesis triggered by chemical signals from tumor cells in a phase of rapid growth. Different factors are known to induce the angiogenesis process. The most important angiogenic factor is Vascular endothelial growth factor (VEGF).

\section{Methods}

Forty histologically confirmed primary OSCC patients and 40 age sex matched controls were included in the study. VEGF gene expression in venous blood was determined using quantitative real time PCR using the TaqMan chemistry. The experiments were done in duplicate keeping the GAPDH gene as the housekeeping gene. Gene expression was determined using the $2-\Delta \Delta C Q$ and fold increment was calculated.

\section{Results}

The mean fold increment of VEGF gene expression among patients were found to be high and showed 2.31 fold increase compared to the control group $(\mathrm{P}<0.001)$. Forty patients comprised of carcinoma of tongue $(28 / 40)$, buccalmucosa $(9 / 40)$, other oral mucosal sites (3/40). Majority [46.42\%(13/28)] OSCC of tongue had fold increment ranging from 2.0-3.0 while 55.55\% (5/9) patient with OSCC in buccal mucosa had the fold increment between 1-1.5. Histologically poorly differentiated cancers $(4 / 40)$ had the fold increment value $>2$.

Moderately differentiated OSCC (8/40) had 2 equal distribution peaks of 1.-1.5 $(5 / 8)$ and 2.-2.5 (3/8). Majority of the patients $77.5 \%(31 / 40)$ were in $\mathrm{T} 1-\mathrm{T} 2$ TNM stage. Among them 23.10\%(9/31) had fold increment of 1.0-1.5, while $33.3 \%(3 / 9)$ of T3-T4 stage had fold increment of 2.5-3.0.

\section{Discussion and Conclusions}

VEGF was significantly upregulated in OSCC patients. Also, these findings suggested a positive correlation between VEGF mRNA expression, TNM stage and histological differentiation. 